\title{
Numerical aspects of two coupled harmonic oscillators
}

\author{
Jihad Asad and Olivia Florea
}

\begin{abstract}
In this study an interesting symmetric linear system is considered. As a first step we obtain the Lagrangian of the system. Secondly, we derive the classical Euler- Lagrange equations of the system. Finally, numerical and analytic solution for these equations have been presented for some chosen initial conditions.
\end{abstract}

\section{Introduction}

As it is known to scientists there are two main approaches that can be used to investigate dynamical systems and obtaining the equations of motion of any system. The Newtonian approach, which is a force (i.e., vector) based one, and an energy approach method (i.e., scalar) invented by the French mathematician Joseph Louis Lagrange. In many cases, we face difficulties in applying the Newtonian approach, since we have to set up all forces acting on the system and sometimes it is not clear. The second approach (i.e, Lagrangian) is a very elegant and useful method for finding the equations of motion for all dynamical systems [1]-[3].

One can see from literature that Lagrangian and Hamiltonian mechanics origin rested on two principles. First they try to express the state of the mechanical system using the minimum representation possible. Second, a mechanical system tries to optimize its action from one split second to the

Key Words: Coupled harmonic Oscillators, Lagrange, Euler- Lagrange equations, numerically solution.

2010 Mathematics Subject Classification: Primary 70H03, 37N30 ; Secondary 37N05.

Received: 28.06.2019

Accepted: 26.07.2019 
next, and as a result the only physical principles the reader has to know are Newtons three laws, the kinetic energy, and the potential energy of the system.

Many important physical systems can be found in many literature review works [1]-[6]. Among these systems we can notice coupled oscillators. Coupled oscillators are oscillators connected in such a way that energy can be transferred between them [1]-[3]. The motion of coupled oscillators can be complex, and does not have to be periodic.

The stability of two dynamical systems using Lyapunov function was studied by Bala in [7]. The structural influence of the forces of the stability of dynamical systems using Hurwitz criterion and also Lyapunov function was studied in [8]. The motion equations of a one-dimensional finite element having a general three-dimensional motion together the body using the Lagranges equations are established in [9]. Similar techniques in order to obtain some theoretical and experimental results can be encountered in papers [15], [16], $[17]$.

Simulink is an environment of Matlab Software that is used for the simulation of dynamical systems and also for the model-based design of embedded systems. With Simulink the considered systems can be designed, simulated and implemented based on a set of block libraries. Different types of continuous, discrete and mixed-signal systems (e.g. communications, controls, signal/video/image processing) are simulated and tested with Simulink, [10][14]. When the mathematical models contain differential or algebraic equations then the behavior of the systems could be explained from the point of view of scientific principles based on the parameters or variables that influence the models over time. In the third part of the present paper the simulation of the considered dynamic system is realized using numerical techniques on the solution of a system of differential equation.

This paper deals with a two coupled harmonic oscillators known in literature as a symmetric linear triatomic molecule. The contribution of authors consists in the analysis of the behavior of the system from a mathematical and also a numerical point of view. The solution of the considered system is obtained analytic using the Laplace transform and the numerical analysis is considered for two modes of oscillations: symmetric and asymmetric using Simulink environment from MATLAB software.

The paper is organized as follows: In sec. 2 a description for the system is presented, where a Lagrange equation is obtained, and as a result the EulerLagrange equations are derived. The mathematical solution is given in the section 3 of the present paper using the Laplace transform. In sec. 4 numerical techniques used are discussed and two cases were considered (anti-symmetric and mixed symmetric). Finally, we close the paper by results and discussion section (sec. 5). 


\section{Description of System}

In this work we are going to investigate a two coupled oscillators. To start, we consider a two coupled harmonic oscillators of three masses connected linearly by two springs each of stiffness $(k)$. The masses at the ends are identical $(m)$, while the mass at the center is differ $(M)$ as indicated in Fig. 1 below. This system is known in literature as a symmetric linear triatomic molecule (e.g, $\left.\mathrm{CO}_{2}\right)$.

The kinetic energy $(T)$, and potential energy $(V)$ of the system respectively are:

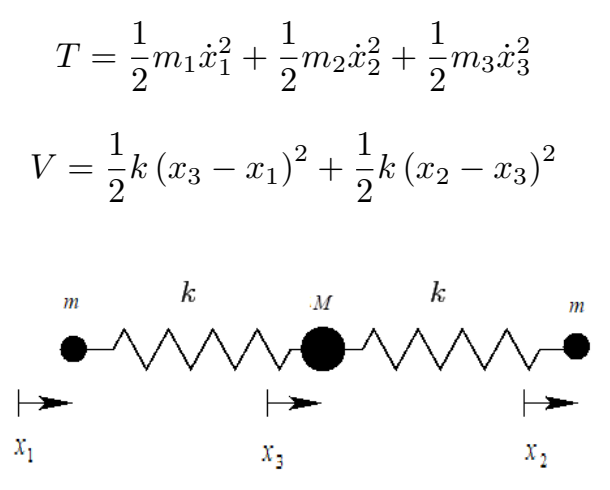

Figure 1: A two coupled harmonic oscillators

As a result the classical Lagrangian reads:

$$
\begin{gathered}
L=T-V \\
L=\frac{1}{2} m_{1} \dot{x}_{1}^{2}+\frac{1}{2} m_{2} \dot{x}_{2}^{2}+\frac{1}{2} m_{3} \dot{x}_{3}^{2}-\frac{1}{2} k\left(x_{3}-x_{1}\right)^{2}-\frac{1}{2} k\left(x_{2}-x_{3}\right)^{2}
\end{gathered}
$$

Now, in order to obtain the classical Euler- Lagrange Equations (CELE's) we use the relation $\frac{\partial L}{\partial x_{i}}-\frac{d}{d t} \frac{\partial L}{\partial \dot{x}_{i}}=0$. With $i=1,2,3$

So for $x_{1}, \quad x_{2}, \quad$ and $x_{3}$ we have

$$
\ddot{x}_{1}=\Omega^{2}\left(x_{3}-x_{1}\right)
$$




$$
\begin{gathered}
\ddot{x}_{2}=\Omega^{2}\left(x_{3}-x_{2}\right) \\
r \ddot{x}_{3}=\Omega^{2}\left(x_{1}+x_{2}-2 x_{3}\right)
\end{gathered}
$$

where $r=\frac{M}{m}$, and $\Omega=\sqrt{\frac{k}{m}}$

It is clear from Eq. (3) that we have 3 degrees of freedom. We can reduce this to 2 degrees of freedom by considering only oscillatory modes of motion (i.e., neglecting translational modes), this can be achieved by demanding that the center of mass of the system remains stationary. Thus, we require that:

$$
m\left(x_{1}+x_{2}\right)+M x_{3}=0
$$

The above equation can be rearranged to give:

$$
x_{3}=-\frac{m}{M}\left(x_{1}+x_{2}\right)
$$

On the other hand

$$
\ddot{x}_{3}=-\frac{m}{M}\left(\ddot{x}_{1}+\ddot{x}_{2}\right)
$$

Now, eliminating $x_{3}$ from Eqs. (4- 6) we yield respectively:

$$
\begin{gathered}
r \ddot{x}_{1}=-\Omega^{2}\left((1+r) x_{1}+x_{2}\right) \\
r \ddot{x}_{2}=-\Omega^{2}\left((1+r) x_{2}+x_{1}\right) \\
\ddot{x}_{1}+\ddot{x}_{2}=\left(1+\frac{2}{r}\right) \Omega^{2} x_{1}+\left(1+\frac{2}{r}\right) \Omega^{2} x_{2}
\end{gathered}
$$

Note here that Eq. (12) is just the sum of Eq. (10) and Eq. (11)

Now, in the next section we aim to solve Eqs. $(10,11)$ numerically for some initial conditions.

\section{Mathematical solution}

In this section we will solve analytic the system formed by Eq. (10) and (11) using the Laplace transform. Therefore the mentioned system can be written in the simplified form:

$$
\left\{\begin{array}{l}
\ddot{x}_{1}=a x_{1}+b x_{2} \\
\ddot{x}_{2}=b x_{1}+a x_{2}
\end{array}\right.
$$

where $a=-\frac{\Omega^{2}(1+r)}{r}$ and $b=-\frac{\Omega^{2}}{r}$.

In a general case we will consider the following initial conditions:

$$
\begin{aligned}
& x_{1}(0)=\alpha_{0} ; \dot{x}_{1}(0)=\alpha_{1} ; \\
& x_{2}(0)=\alpha_{2} ; \dot{x}_{2}(0)=\alpha_{3} ;
\end{aligned}
$$


Using the property of original derivation and noted by $\mathcal{L}$ the Laplace transform we have:

$$
\begin{array}{r}
\mathcal{L}\left[x_{1}(t)\right](s)=X_{1}(s) ; \quad \mathcal{L}\left[x_{2}(t)\right](s)=X_{2}(s) ; \\
\mathcal{L}\left[\ddot{x}_{1}(t)\right](s)=s^{2} X_{1}(s)-s \alpha_{0}-\alpha_{1} ; \\
\mathcal{L}\left[\ddot{x}_{2}(t)\right](s)=s^{2} X_{2}(s)-s \alpha_{2}-\alpha_{3} ;
\end{array}
$$

Thus the system (13) will have the following form:

$$
\left\{\begin{array}{c}
\left(s^{2}-a\right) X_{1}(s)-b X_{2}(s)=s \alpha_{0}+\alpha_{1} \\
-b X_{1}(s)+\left(s^{2}-a\right) X_{2}(s)=s \alpha_{2}+\alpha_{3}
\end{array}\right.
$$

Solving the above system we will obtain the images through the Laplace transform:

$$
X_{1}(s)=\frac{s^{3} \alpha_{0}+s^{2} \alpha_{1}+s\left(b \alpha_{2}-a \alpha_{0}\right)+b \alpha_{3}-a \alpha_{1}}{\left[s^{2}+\xi^{2}\right]\left[s^{2}+\zeta^{2}\right]}
$$

respectively,

$$
X_{2}(s)=\frac{s^{3} \alpha_{2}+s^{2} \alpha_{3}+s\left(b \alpha_{0}-a \alpha_{2}\right)+b \alpha_{1}-a \alpha_{3}}{\left[s^{2}+\xi^{2}\right]\left[s^{2}+\zeta^{2}\right]}
$$

where $\xi^{2}=-a-b=\frac{\Omega^{2}}{r}(2+r) ; \zeta^{2}=-a+b=\Omega^{2}$. Using the inverse method of Mellin Fourier for Laplace transform we will deduce the originals:

$$
x_{1}(t)=A_{1} \cos (\zeta t)+\frac{B_{1}}{\zeta} \sin (\zeta t)+C_{1} \cos (\xi t)+\frac{D_{1}}{\xi} \sin (\xi t)
$$

where $A_{1}=\frac{\alpha_{0}-\alpha_{2}}{2} ; B_{1}=\frac{\alpha_{1}-\alpha_{3}}{2} ; C_{1}=\frac{\alpha_{2}+\alpha_{0}}{2} ; D_{1}=\frac{\alpha_{1}+\alpha_{3}}{2}$, respectively

$$
x_{2}(t)=A_{2} \cos (\zeta t)+\frac{B_{2}}{\zeta} \sin (\zeta t)+C_{2} \cos (\xi t)+\frac{D_{2}}{\xi} \sin (\xi t)
$$

where $A_{2}=\frac{\alpha_{2}-\alpha_{0}}{2} ; B_{2}=\frac{\alpha_{3}-\alpha_{1}}{2} ; C_{1}=\frac{\alpha_{2}+\alpha_{0}}{2} ; D_{1}=\frac{\alpha_{1}+\alpha_{3}}{2}$.

We observe that $A_{1}=-\stackrel{A}{2}_{2}, B_{1}=-B_{2}, C_{1}=C_{2}, D_{1}=D_{2}$.

\section{Numerical solution}

The system presented in our work has two modes of oscillations. The first mode of oscillation occurred when $x_{1}(0)=-x_{2}(0)$, and $x_{3}(0)=0$. This mode of oscillation is known in literature as symmetric (breathing) mode. In this mode of oscillation, the two end atoms move in opposite direction whilst the central atom remains stationary. 
The second mode in our system is the asymmetric mode in which $x_{1}(0)=$ $x_{2}(0)$, and $x_{3}(0)=\frac{-2}{r} x_{1}(0)=\frac{-2}{r} x_{2}(0)$. In this mode of oscillation the two end atoms move in the same direction whilst the central atom moves in the opposite direction.

Below, we aim to study the above two modes numerically for two cases of coupled oscillators. In the first case (i.e., case I) we consider $r=\frac{M}{m}=1.50$, with the following values of angular frequencies $\Omega=\sqrt{\frac{k}{m}}=0.50,1.00$, and 1.50, while for the second case(i.e., case II) with the following values of angular frequencies $\Omega=\sqrt{\frac{k}{m}}=0.50,1.00$, and 1.50 .

Therefore, our system of differential equations will have the following form

$$
\left\{\begin{array}{c}
x_{1}^{\prime \prime}=a x_{1}+b x_{2} \\
x_{2}^{\prime \prime}=b x_{1}+a x_{2} \\
x_{3}^{\prime \prime}=c\left(x_{1}+x_{2}\right)
\end{array}\right.
$$

where $a=-\frac{\Omega^{2}(1+r)}{r}, b=-\frac{\Omega^{2}}{r}, c=\frac{\Omega^{2}(2+r)}{r}$.

The numerical analysis is realized using the Simulink environment from Matlab. The Simulink scheme of the system (13) is:

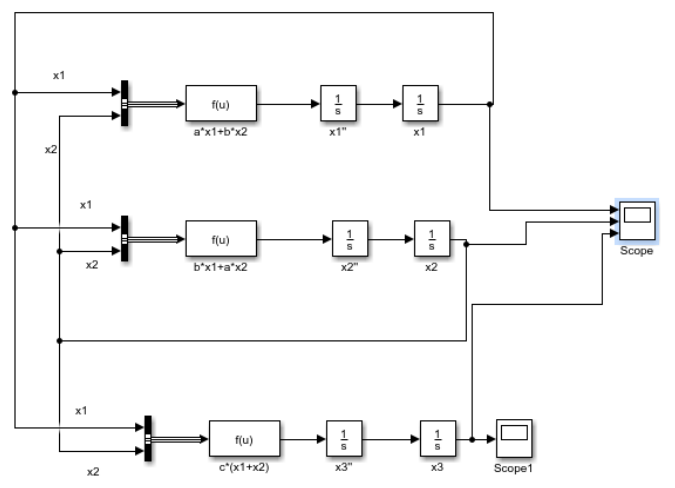

Figure 2: The Simulink scheme for the system (13) using the Fcn block from the block library User Defined Functions

\subsection{The symmetric mode}

For this mode of oscillation let us assume that $x_{1}(0)=-x_{2}(0)=1$, and $x_{3}(0)=0$, while $\dot{x}_{1}(0)=\dot{x}_{2}(0)=\dot{x}_{3}(0)=0$. 


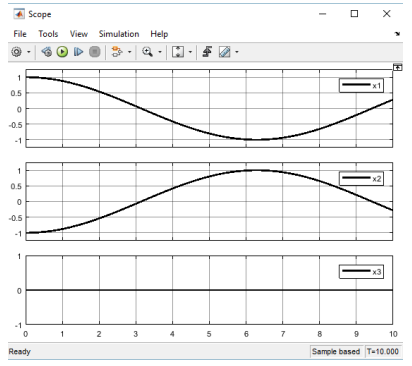

Figure 3: The behavior of the solutions of the system (13) in the case when $\mathrm{r}=1.5$ and $\Omega=\sqrt{\frac{k}{m}}=0.50$

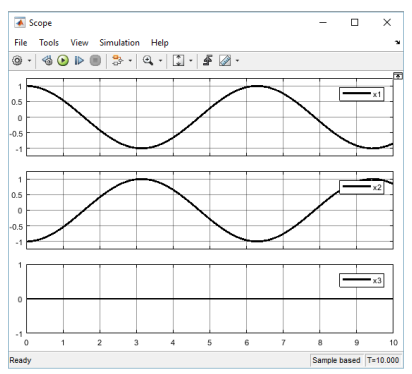

Figure 4: The behavior of the solutions of the system (13) in the case when $\mathrm{r}=1.5$ and $\Omega=1.0$ 


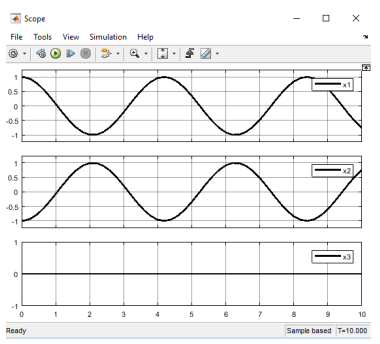

Figure 5: The behavior of the solutions of the system (13) in the case when $\mathrm{r}=1.5$ and $\Omega=1.5$

\subsection{The asymmetric mode}

For this mode $x_{1}(0)=x_{2}(0)=1$, and $x_{3}(0)=\frac{-2}{r} x_{1}(0)=\frac{-2}{r} x_{2}(0)=\frac{-2}{r}$. While $\dot{x}_{1}(0)=\dot{x}_{2}(0)=\dot{x}_{3}(0)=0$.

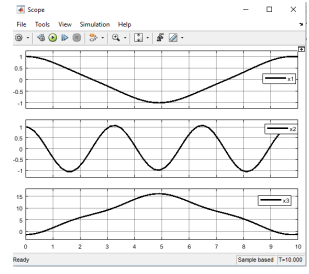

Figure 6: The behavior of the solutions of the system (13) in the case when $\mathrm{r}=1.5$ and $\Omega=\sqrt{\frac{k}{m}}=0.50$

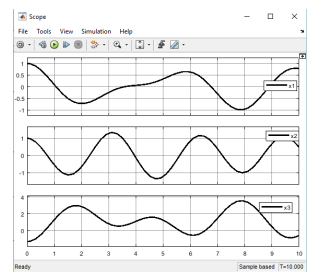

Figure 7: The behavior of the solutions of the system (13) in the case when $\mathrm{r}=1.5$ and $\Omega=1.00$ 


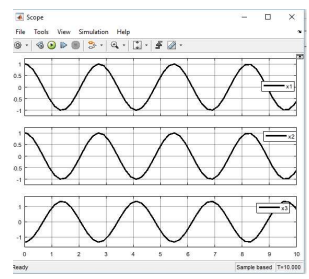

Figure 8: The behavior of the solutions of the system (13) in the case when $\mathrm{r}=1.5$ and $\Omega=1.5$

\section{$5 \quad$ Results and Discussion}

In this study, we investigated numerically the behaviour of a famous physical system called a linear two coupled oscillators. This intresting system is a symmetric linear system, with carbon dioxide $\mathrm{CO}_{2}$ being a good example. Firstly, the symmetric mode, where the behaviour of the system has been presented in Figs. 3- 5. Secondly, the asymmetric, where the behaviour of the system has been showed in Figs. 6- 8 .

The numerical analysis of the system (13) is realized using the Simulink environment, part of MATLAB software. Due to the fact that the differential equations from (13) are of second degree we used two integrator blocks that are continuous-time integration of the input signal with initial conditions of internal type in order to obtain the behaviour of the solutions. To simplify the Simulink scheme, the expressions of those three equations are inserted in three different function blocks. The scheme is realized based on the loop diagrams, therefore in front of the function blocks we included a bus creator that creates a bus signal from its inputs.

Figures 3- 5 show the behaviour of the solutions of the system represented by Eq. (13) for different values of $\Omega$. It is clear from these figures that the particle at the center remains at rest as we assumed previously, while the position of mass 1 behaves in the opposite direction to that of particle 2 . This is why we call this mode symmetric. Also the resulting motion of masses 1 , and 2 is oscillatory motion but with $180^{\circ}$ out of phase with each other.

In figs. 6- 8, the behaviour of the solutions of the system represented by Eq. (13) for different values of $\Omega$ has been showed. For this asymmetric mode it is clear from Figs. 6- 8 that the behaviour of the solution of the system (13) depends on the value of $\Omega$. For example, Fig. 8 shows that we have oscillatory motion for the three masses but as cleare mass 3 with $180^{\circ}$ out of phase with the other two masses. 


\section{References}

[1] J.B. Marion, S.T. Thornton, Classical Dynamics of Particles and Systems, 5th Edition, Brooks Cole (2003)

[2] W. Greiner, Classical Mechanics, Systems of Particles and Hamiltonian Dynamics, Springer-Verlag Berlin Heidelberg (2010)

[3] Goldstein H, Poole C P, and Safko J L, Classical Mechanics, 3rd edn. Addison Wesley (1980).

[4] N.E. Martnez-Prez, C. Ramrez, On the Lagrangian description of dissipative systems, Journal of Mathematical Physics, 59 (3) (2018).

[5] D.M. Gitman, V.G. Kupriyanov, Canonical quantization of so-called nonLagrangian systems, European Physical Journal, 50 (3), (2007).

[6] C.P. Pesce, The Application of Lagrange Equations to Mechanical Systems With Mass Explicitly Dependent on Position, Journal of Applied Mechanics. 70, (2007), 751- 756.

[7] D. Bala, Geometric Methods In Study Of The Stability Of Some Dynamical Systems, An. St. Univ. Ovidius Constanta, 17(3, (2009), 27-35.

[8] M. Lupu, O. Florea, C. Lupu, The structural influence of the forces of the stability of dynamical systems, An. St. Univ. Ovidius Constanta 17(3), (2009), 159 - 169.

[9] S. Vlase, M. Marin, A. Ochsner, ML Scutaru, Motion equation for a flexible one-dimensional element used in the dynamical analysis of a multibody system, Continuum Mechanics and Thermodynamics, 31(3), (2019), 715724 .

[10] D. Dingy Xue, Y. Chen, System Simulation Techniques with MATLAB and Simulink, Wiley, (2013)

[11] H. Klee, R. Allen, Simulation of Dynamic Systems with MATLAB and Simulink, CRC Press Taylor \& Francis Group, (2011)

[12] D. K. Chaturvedi, Modeling and Simulation of Systems Using MATLAB and Simulink, CRC Press Taylor \& Francis Group, (2010)

[13] O. Beucher, M. Weeks, Introduction to MATLAB and SIMULINK, A Project Approach, Third Edition (Engineering, Infinity Science Press LLC Hingham, Massachusetts New Delhi, (2006) 
[14] H-P Halvorsen, Introduction to Simulink, Faculty of Technology, Norway, http://www.academia.edu/9207393/Introduction_to_Simulink

[15] Gh. Lupu, E.M. Craciun, E. Suliman, An Extension of Equilibrum Problem for the Plane Simple Pendulum, An. St. Univ. Ovidius Constanta, seria Matematica, 1, (1993), 141-145.

[16] Gh. Lupu, A. Rabaea, E.M. Craciun, Theoretical Aspects Concerning Vibrations of Elastical Technological Systems, An. St. Univ. Ovidius Constanta, seria Matematica, 1, (1993), 133-139.

[17] G. Groza, A-M Mitu, N. Pop, T. Sireteanu, Transverse vibrations analysis of a beam with degrading hysteretic behavior by using Euler-Bernoulli beam model, An. St. Univ. Ovidius Constanta, seria Matematica, 26(1), (2018), $125-139$.

Jihad ASAD,

Department of Physics,

College of Sciences, Palestine Technical University (PTUK)

P.O. Box 7, Tulkarm, Palestine

Tel.: +123-45-678910

Fax: +123-45-678910

Email: drjasad@yahoo.com

Olivia FLOREA

Department of Mathematics and Computer Science,

Transilvania University of Brasov, Romania

Email: olivia.florea@unitbv.ro 